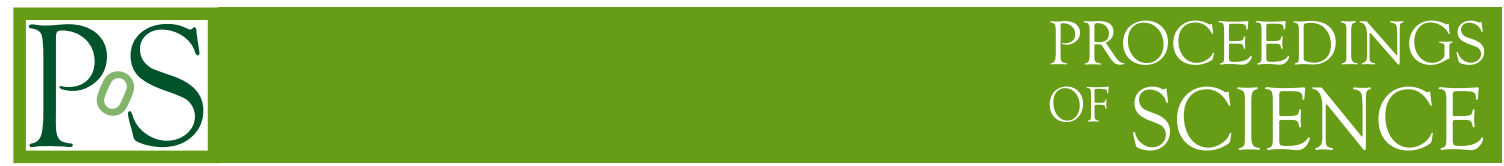

\title{
Measurement of strong-phase parameters at BESIII
}

\section{Anita Lavania ${ }^{a, *}$}

${ }^{a}$ Inidian Institute of Technology Madras, Chennai, India

E-mail: anitalavania@physics.iitm.ac.in

BESIII has collected $2.9 \mathrm{fb}^{-1}$ of $e^{+} e^{-}$collision data at $3.773 \mathrm{GeV}$. We report recent measurements of strong-phase differences in $D^{0}$ and $\bar{D}^{0}$ decays of $K_{\mathrm{S}}^{0} \pi^{+} \pi^{-}, K_{\mathrm{S}}^{0} K^{+} K^{-}, K^{-} \pi^{+} \pi^{-} \pi^{+}$and $K^{-} \pi^{+} \pi^{0}$. The obtained parameters are important to reduce the systematic uncertainty in the $\gamma / \phi_{3}$ measurement at LHCb and Belle II.

*** The European Physical Society Conference on High Energy Physics (EPS-HEP2021), *** *** 26-30 July 2021 ***

*** Online conference, jointly organized by Universität Hamburg and the research center DESY $* * *$

${ }^{*}$ Speaker 


\section{Objective}

Determining the elements of Cabibbo-Kobayashi-Maskawa (CKM) matrix [1] describes charged weak currents, and over-constraining its unitarity triangles provide an open-ground for better understanding of $\mathcal{C P}$ violation and presents a unique opportunity to test physics beyond Standard Model. The parameter $\gamma$ or $\phi_{3}$ is, upto third order in the sine of the Cabibbo-angle, approximated as the argument of the CKM matrix element $V_{u b}$. It is accessible experimentally, indirectly through loop and penguin level processes e.g. $B^{0} \rightarrow J / \psi K_{\mathrm{S}}^{0}$ and $B^{0}-\bar{B}^{0}$ mixing, as well as directly in relatively low branching fraction tree-level $B^{ \pm} \rightarrow D K^{ \pm}$decay, involving neutral $D$ mesons decaying in a superposed state. In such a direct measurement of $\gamma$, charm hadronic parameters are an inevitable source of systematic uncertainty and will be the most dominating ones once $\gamma$ reaches the desired $\sim 1^{\circ}$ precision level [2]. Furthermore, these parameters are also essential for model-independent determination of charm mixing parameters and search for $\mathcal{C P}$ violation in $D \rightarrow K_{\mathrm{S}}^{0} \pi^{+} \pi^{-}$decays [3]. These proceedings summarize the most precise measurements performed on these hadronic charm inputs at BESIII for the decay modes $K^{0} \pi^{+} \pi^{-}, K^{0} K^{+} K^{-}, K^{-} \pi^{+} \pi^{-} \pi^{+}$, and $K^{-} \pi^{+} \pi^{0}$.

\section{Strong-phase parameters in $D$ decay}

The hadronic parameters associated with the interference in $D^{0}$ and $\bar{D}^{0}$ decays contributing coherently towards a single final state $\mathcal{S}$, are indicated below in an integrated $B$ decay rate

$$
\Gamma\left(B^{-} \rightarrow D K^{-}\right) \propto\left(r_{D}^{\mathcal{S}}\right)^{2}+r_{B}^{2}+2 r_{B} r_{D}^{\mathcal{S}} R_{D} \cos \left(\delta_{B} \mp \gamma-\Delta \delta_{D}^{\mathcal{S}}\right)
$$

where $r_{D}^{\mathcal{S}}$ is the ratio of amplitudes between doubly-Cabibbo-suppressed (DCS) and Cabibbofavored $(\mathrm{CF}) D$ decays, and $R_{D}^{\mathcal{S}} e^{-i \Delta \delta_{D}^{\mathcal{S}}}$ characterizes the interference, integrated over the phase-space of the final state. The magnitude $R_{D}^{\mathcal{S}}$ is called the average coherence factor and $\Delta \delta_{D}^{\mathcal{S}}$ is the strong-phase difference between the flavor state $D$ decays [4]:

$$
R_{D}^{\mathcal{S}} e^{-i \Delta \delta_{D}^{\mathcal{S}}}=\left\langle\mathcal{A}_{D}^{\mathcal{S}} \overline{\mathcal{A}}_{D}^{\mathcal{S} *}\right\rangle=\frac{\int \mathcal{A}_{D}^{\mathcal{S}}(\mathbf{x}) \overline{\mathcal{A}}_{D}^{\mathcal{S} *}(\mathbf{x}) d \mathbf{x}}{A_{D}^{\mathcal{S}} A_{\bar{D}}^{\mathcal{S}}},
$$

where, $\mathcal{A}_{D}^{\mathcal{S}}\left(\overline{\mathcal{A}}_{D}^{\mathcal{S}}\right)$ is the $D^{0}\left(\bar{D}^{0}\right)$ decay amplitudes at position $\mathrm{x}$ on the $\mathcal{S}$ phase-space and $A_{D}^{\mathcal{S}}, A_{\bar{D}}^{\mathcal{S}}$ are the total integrated amplitudes. These three parameters, $r_{D}^{\mathcal{S}}, R_{D}^{\mathcal{S}}$, and $\Delta \delta_{D}^{\mathcal{S}}$ are typically collectively referred to as strong-phase parameters. The parameters $R_{D}$ and $\Delta \delta_{D}$ may be represented in various forms depending on the final decay state and the corresponding analysis technique. Specific details on the definition of observables used for the four decay modes are included in the next section.

\section{Measurement at BESIII}

At BESIII, pairs of $D \bar{D}$ mesons are produced at threshold, which approximately corresponds to the mass of the $\psi(3770)$ resonance, and are quantum-correlated by anti-symmetry 
of the total wavefuntion with charge conjugation quantum number constrained to one, resulting in very powerful, almost exact tagging ( $C P$ and flavor) capabilities in a $\sim 93 \%$ hermetic detector. No additional particles are produced apart from the $D \bar{D}$ pair leading to a clean environment. This allows measurements of the strong-phase parameters when the $D$ mesons are reconstructed in the required signal decay modes. The rate of a typical $\psi(3770) \rightarrow D(\rightarrow \mathcal{S}) \bar{D}(\rightarrow \mathcal{T})$ decay chain is given by

$$
\Gamma_{\mathcal{S} \mathcal{T}}=|\langle\mathcal{S} \mathcal{T} \mid \psi\rangle|^{2} \propto\left(r_{D}^{\mathcal{S}}\right)^{2}+\left(r_{\bar{D}}^{\mathcal{T}}\right)^{2}-2 R_{D}^{\mathcal{S}} R_{D}^{\mathcal{T}} r_{D}^{\mathcal{S}} r_{\bar{D}}^{\mathcal{T}} \cos \left(\Delta \delta_{D}^{\mathcal{S}}-\Delta \delta_{D}^{\mathcal{T}}\right)
$$

Analysis techniques and key results for the mentioned hadronic decay modes are summarized in the subsections below.

\section{$3.1 K_{\mathrm{S} / \mathrm{L}}^{0} h^{+} h^{-},(h=\pi, K)$}

The three-body self-conjugate final states $K_{\mathrm{S} / \mathrm{L}}^{0} \pi^{+} \pi^{-}$and $K_{\mathrm{S} / \mathrm{L}}^{0} K^{+} K^{-}$proceed through a rich variety of sub-processes and therefore the total decay amplitudes possess non-trivial resonant substructures on the two-dimensional phase-space $\left(s_{K_{\mathrm{S} / \mathrm{L}}^{0} h^{+}}, s_{K_{\mathrm{S} / \mathrm{L}}^{0} h^{-}}\right)$of the final decay products, known as a Dalitz plot. Therefore, instead of using an average value for the strong-phase parameters over the entire phase-space, the Dalitz-plot is binned into various bins subject to minimum variation in $\Delta \delta_{D}$ within individual bins and sensitivity to $\gamma$ [6]. The parameters of interest are cosines and sines of strong-phase differences, averaged out in the individual bins, $i$ of the Dalitz-plot [5]:

$$
c_{i}\left(s_{i}\right)=\frac{\int_{D_{i}}\left|\mathcal{A}_{D}\right|\left|\overline{\mathcal{A}}_{D}\right| \cos (\sin ) \Delta \delta_{D} d \mathbf{x}}{\sqrt{\int_{D_{i}}\left|\mathcal{A}_{D}\right|^{2} d \mathbf{x} \int_{D_{i}}\left|\overline{\mathcal{A}}_{D}\right|^{2} d \mathbf{x}}} .
$$

The central idea is to reconstruct signal $D \rightarrow K_{\mathrm{S} / \mathrm{L}}^{0} h^{+} h^{-}(h=\pi, K)$ events tagged by states with known $C P$ content, e.g. $K^{+} K^{-}$or $\pi^{+} \pi^{-} \pi^{0}$, and by a $K_{\mathrm{S}}^{0} h^{+} h^{-}$state, the yields of which are the observables in these analyses and are sensitive to $c_{i}$ and $s_{i}$. Likelihood functions consisting of Poisson probability density functions in double-tag yields for various tag modes are minimized simultaneously to extract $c_{i}$ and $s_{i}$. In these analyses, an

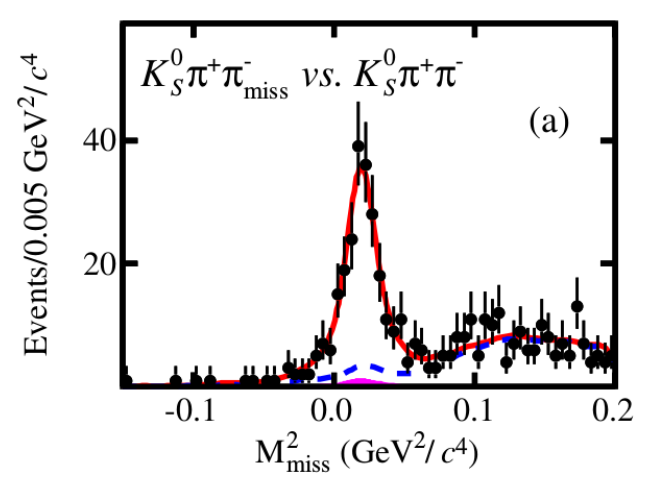

Figure 1: Missing mass distribution in $K_{\mathrm{S}}^{0} \pi^{+} \pi^{-}$vs. $K_{\mathrm{S}}^{0} \pi^{+} \pi^{-}$double-tag events with one pion as missing track.

approximately three times larger dataset than the previous analysis from CLEO is used. In 
addition, extra $C P$ tag modes, $\pi^{+} \pi^{-} \pi^{0}, K_{\mathrm{L}}^{0} \pi^{0} \pi^{0}$, are incorporated that provide more constraints. Furthermore, to achieve improved efficiencies, partial reconstruction techniques on some multibody tag modes are employed in the $K_{\mathrm{S}}^{0} \pi^{+} \pi^{-}$analysis as shown in Fig. 1. The $c_{i}, s_{i}$ results for the two signal modes are shown in Fig. 2. The propagated uncertainty on $\gamma$ from the $K_{\mathrm{S}}^{0} \pi^{+} \pi^{-}$and $K_{\mathrm{S}}^{0} K^{+} K^{-}$decay modes are expected to be $1.2^{\circ}$ and $2.4^{\circ}$ respectively $[7,8]$.
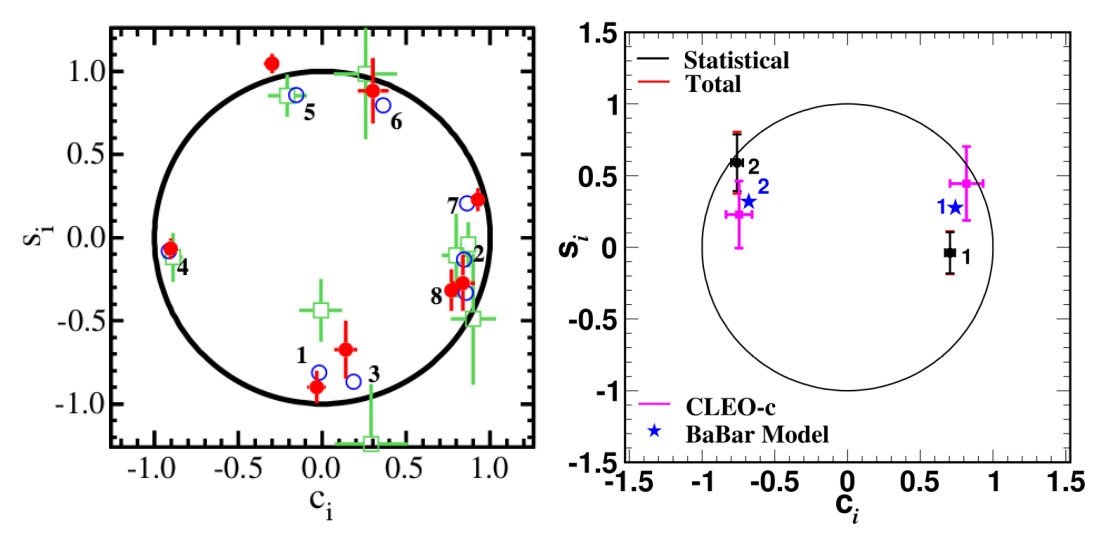

Figure 2: Measured model-independent $c_{i}, s_{i}$ values for $K_{\mathrm{S}}^{0} \pi^{+} \pi^{-}$(optimal binning) (left) and $K_{\mathrm{S}}^{0} K^{+} K^{-}$(equal $\Delta \delta_{D}$ binning) (right) modes compared against the 2010 CLEO results and the 2008 BABAR model-dependent values [7, 8].

\section{$3.2 \quad K^{-} \pi^{+} \pi^{-} \pi^{+}$and $K^{-} \pi^{+} \pi^{0}$}

The parameters of interest for the flavor state decay modes $K^{-} \pi^{+} \pi^{-} \pi^{+}$and $K^{-} \pi^{+} \pi^{0}$ are the average coherence factors $R_{D}^{\mathcal{S}}$ and strong-phase difference $\Delta \delta_{D}^{\mathcal{S}}$. However, in this analysis strong-phase parameters are also measured in bins of the five-dimensional phasespace of the signal mode $K^{-} \pi^{+} \pi^{-} \pi^{+}$. CP specific states, flavor states with same kaon flavor as that on the signal side (like-sign, LS) and mixed $C P$ state $\left(K_{\mathrm{S}}^{0} \pi^{+} \pi^{-}\right)$are used as tag modes. The observables for strong-phase calculation with the signal versus $K_{\mathrm{S}}^{0} \pi^{+} \pi^{-}$ tag mode are simply the double-tag yields. For the rest of the tag modes the observables are ratios of the measured yields to the expected yields under no quantum-correlation hypothesis, denoted by $\rho_{C P}^{\mathcal{S}, \mathcal{T}}$ and $\rho_{L S}^{\mathcal{S}, \mathcal{T}}$. Measurements on these observables can be made using branching-fraction information of the signal and tag modes for the incoherent normalization together with the double-tagged signal yields. The functions outlined in the subsections below express the $\rho$ observables in terms of the average strong-phase parameters.

\section{$C P$ tag}

For the $C P$ tag modes, the parameters $r_{D}^{\mathcal{T}}, R_{D}^{\mathcal{T}}$ and $\Delta \delta_{D}^{\mathcal{T}}$ are 1,1 and 0 respectively. Therefore, the expected $\rho_{C P}$ observables are given by,

$$
\rho_{C P}^{\mathcal{S}, \mathcal{T}}=1-\left(2 \mathcal{F}_{C P+}^{\mathcal{T}}-1\right) \frac{2 R_{D}^{\mathcal{S}} r_{D}^{\mathcal{S}} \cos \left(\Delta \delta_{D}^{\mathcal{S}}\right)}{1+\left(r_{D}^{\mathcal{S}}\right)^{2}},
$$

where, $\mathcal{F}_{C P+}^{\mathcal{T}}$ is the $C P$ even content of the tag mode. It is to be noted that due to limited precision on various $C P$ tag decay mode branching fractions, the incoherent normalization 
part in the calculation of the observed value is manipulated to be written in terms of the $D \rightarrow K \pi$ branching fraction and $K \pi v s$. $C P$ double-tag yields. Departure from the nominal value of one for all the $C P$ tags indicates significant coherence.
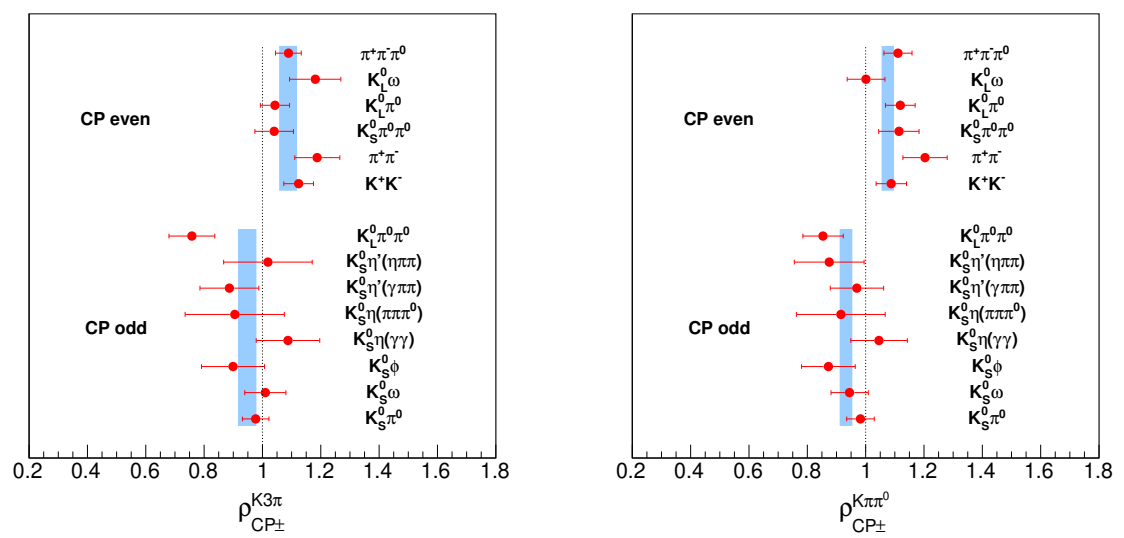

Figure 3: Observed $\rho_{C P}^{\mathcal{S}, \mathcal{T}}$ for $\mathcal{S}=K^{-} 3 \pi$ (left) and $\mathcal{S}=K^{-} \pi^{+} \pi^{0}$ (right) overlayed on the incoherent value of 1 . Blue bands indicate $\pm 1 \sigma$ of the average values for each of the four observables [9].

\section{Like-sign flavor tag}

These are the tag modes that have kaons of the same flavor as that on the signal side, for example $K^{-} \pi^{+}$vs. $K^{-} 3 \pi$ and $K^{-} 3 \pi$ vs. $K^{-} 3 \pi$. Coherence features are significantly more dominant in the same sign decay topologies as compared to the CF opposite sign decays. As an elementary example, the expected $\rho_{L S}$ observable for the decay type $K^{-} 3 \pi v s . K^{-} 3 \pi$ is given by,

$$
\rho_{L S}^{K^{-} 3 \pi, K^{-} 3 \pi}=1-\left(R_{D}^{K^{-} 3 \pi}\right)^{2} .
$$

For the normalization in the calculation of $\rho_{L S}$ observed values, the CF opposite-sign double tag yields are used for their large statistics.

$K_{\mathrm{S}}^{0} \pi^{+} \pi^{-}$tag

Extensive strong-phase parameter studies on $K_{\mathrm{S}}^{0} \pi^{+} \pi^{-}$decay mode have been performed and the latest is also reviewed in these proceedings. The observables are just the double tag yields $\left(Y_{i}\right)$ in bins $(i)$ of $K_{\mathrm{S}}^{0} \pi^{+} \pi^{-}$phase-space as mentioned in Sec. 3.1. The expected yields as functions of the strong-phase parameters are given by

$$
Y_{i}=h\left(K_{i}+\left(r_{D}^{\mathcal{S}}\right)^{2} K_{-i}-2 r_{D}^{\mathcal{S}} R_{D}^{\mathcal{S}} \sqrt{K_{i} K_{-i}}\left(c_{i} \cos \Delta \delta_{D}^{\mathcal{S}}-s_{i} \sin \Delta \delta_{D}^{\mathcal{S}}\right)\right)
$$

A $\chi^{2}$ fit is performed with all the functional observables associated with $C P$, like-sign and $K_{\mathrm{S}}^{0} \pi^{+} \pi^{-}$tag modes constrained to their measured values. The fit results, i.e. $R_{D}$ and $\Delta \delta_{D}$, are shown in Fig. 4 that suggest significant reduction of the allowed regions, especially in $3 \sigma$. A similar $\chi^{2}$ analysis is also carried out in bins of $K^{-} \pi^{+} \pi^{-} \pi^{+}$phase-space bins and the hadronic parameter results are shown in Fig. 5. 

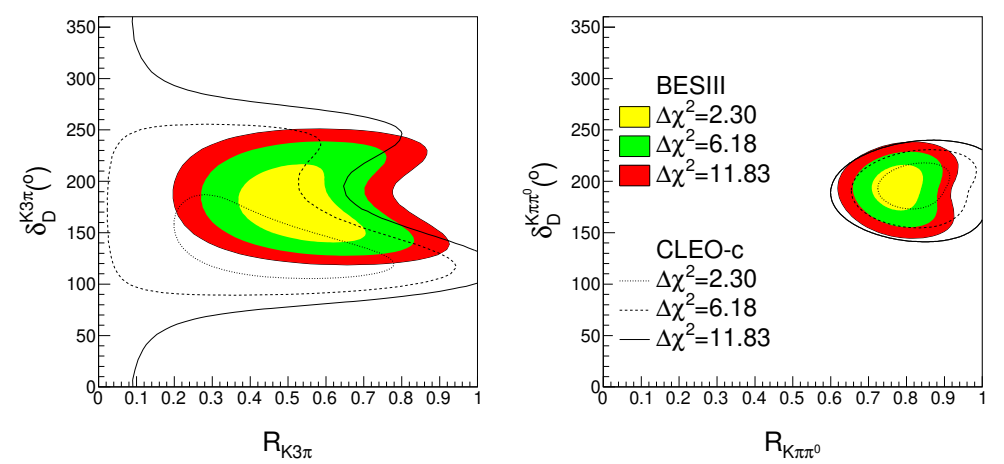

Figure 4: $\Delta \chi^{2}$ scans on average $R_{D}, \Delta \delta_{D}$ fits compared with the previous CLEO results [9].
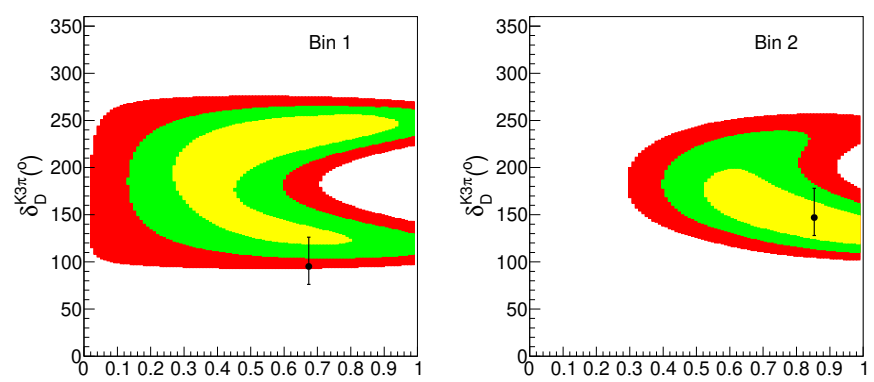

$\mathrm{R}_{\mathrm{K} 3 \pi}$
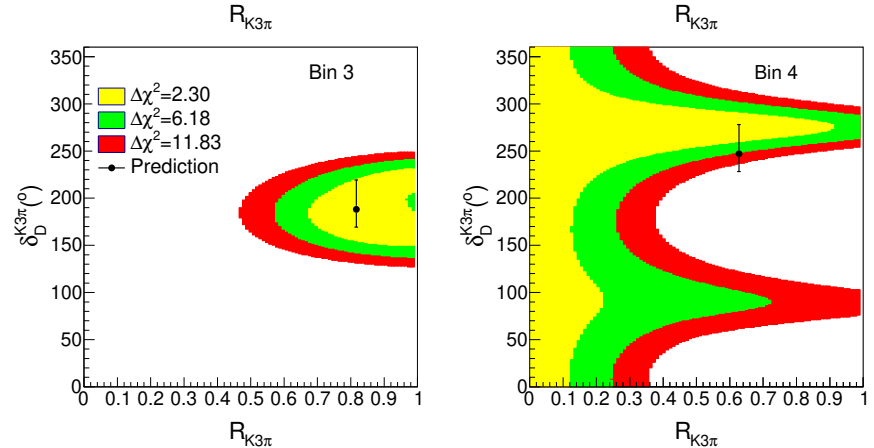

Figure 5: $\Delta \chi^{2}$ scans on $R_{D}, \Delta \delta_{D}$ fits in bins of $K^{-} 3 \pi$ phase-space [9].

\section{Summary}

With $2.93 \mathrm{fb}^{-1}$ of $D \bar{D}$ dataset at BESIII, updated and more precise measurements on strong-phase hadronic parameters for the decay modes $K_{\mathrm{S}}^{0} \pi^{+} \pi^{-}, K_{\mathrm{S}}^{0} K^{+} K^{-}, K^{-} 3 \pi$ and $K^{-} \pi^{+} \pi^{0}$ are presented and these results shrink the charm input systematic uncertainty onto $\gamma$ upto $1.2,2.4$ and $\left({ }_{-7}^{+5}\right)^{\circ}$ degrees [7-9], respectively.

\section{References}

[1] N. Cabibbo, Phys. Rev. Lett. 10, 531 (1963); M. Kobayashi and T. Maskawa, Prog. Theor. Phys. 49, 652 (1973).

[2] D. King, M. Kirk, A. Lenz and T. Rauh, JHEP 03 (2020) 112 [arXiv:1911.07856] [INSPIRE]. 
[3] The LHCb Collaboration, Phys. Rev. Lett. 127111801 (2021).

[4] D. Atwood and A. Soni, Phys. Rev. D 68033003 (2003).

[5] The CLEO collaboration, Phys. Rev. D 82, 112006 (2010)

[6] A. Giri, Y. Grossman, A. Soffer, and J. Zupan, Phys. Rev. D 68, 054018 (2003).

[7] The BESIII Collaboration, Phys. Rev. D 101, 112002 (2020).

[8] The BESIII Collaboration, Phys. Rev. D 102, 052008 (2020).

[9] The BESIII collaboration, J. High Energy Phys. 164 (2021). 